\title{
STRUCTURAL SIZING OF A ROTORCRAFT FUSELAGE USING AN INTEGRATED DESIGN APPROACH
}

\author{
Dominik B. Schwinn*, Peter Weiand ${ }^{* *}$, Matthias Schmid ${ }^{* * *}$ and Michel Buchwald** \\ German Aerospace Center (DLR) \\ ${ }^{*}$ Institute of Structures and Design, \\ ** Institute of Flight Systems, \\ *** Institute of Aerodynamics and Flow Technology
}

\begin{abstract}
Keywords: rotorcraft integrated design; multidisciplinary design optimization; airframe structural sizing; weight estimation
\end{abstract}

\begin{abstract}
Many years the primary design objective of new helicopters was the design of the main rotor(s). Within the last couple of years, this approach has changed into an assessment of all helicopter components as an overall system, thus turning rotorcraft design into a highly interdisciplinary process. Aerodynamics, flight mechanics, structural evaluation, etc. strongly affect each other and these mutual influences are taken into account from the very first design stages. However, weight prediction in early stages still represents an essential part of the design process as it determines the basic properties of the rotorcraft. Due to its function to carry crew and payload as well as to serve as the central mounting for all components, the fuselage represents a major part of the rotorcraft, therefore the structural design of the fuselage airframe constitutes a significant factor of the rotorcraft design at preliminary level.
\end{abstract}

\section{Introduction}

Rotorcraft design is a highly challenging discipline within the aeronautical sciences. Like aircraft design it is, in general, classified into three consecutive phases: The conceptual, the preliminary, and the detailed design phase.

Conceptual design is mainly concerned with the outer configuration of the helicopter, i.e. the aerodynamic shape (loft), rotor characteristics, flight performance analysis, etc. using fast analytical simplified methods or estimations based on statistics, if available.

The subsequent preliminary design phase uses higher fidelity tools to increase the detail level. During this stage a basic internal arrangement is elaborated, i.e. the distribution of primary structure within the previously determined loft. Flight and ground load cases are evaluated, thus enabling the designers to derive the main load paths and to obtain major loads and stresses within the structure.

Ultimately, the detailed design phase is concerned with detailed local solutions, such as joints and fittings. This phase is highly influenced by producibility and maintainability aspects. However, despite this stage being conducted immediately before initializing the manufacturing process, most of the costs are already determined during the conceptual and preliminary design stage [17], thus highlighting the importance of early design phases.

For many years the design of a new helicopter was mainly synonymous to the design of the main rotor(s). However, in recent years the design objective has shifted into an overall examination of all helicopter components as a complete, global system [24]. As an example, the aerodynamics of the fuselage has received considerable attention lately and has become an important part of the de- 
sign process [10]. Since aerodynamics, flight mechanics, structures, etc. work together, helicopter design has become a highly interdisciplinary process.

In order to assess novel configurations addressing typical rotorcraft limitations, e.g. cruise speed, the German Aerospace Center (DLR) started in 2010 with the set-up of an automated, integrated, parametric, and multidisciplinary process chain for early helicopter design. During the projects RIDE (Rotorcraft Integrated Design and Evaluation) and EDEN (Evaluation and Design of Novel Rotorcraft Concepts) the data format CPACS (Common Parametric Aircraft Configuration Schema, [12]) was adapted to match parametric rotorcraft description. The network based simulation environment RCE (Remote Component Environment, [22]) was used to generate and set up workflows to design generic rotorcraft concepts according to user specified top level aircraft requirements (TLARs), typically consisting of payload, cabin volume, range and cruise speed.

During the conceptual branch of the aforementioned process chain the geometry of the desired rotorcraft is generated to fulfill the specified TLARs. At this stage component masses are estimated using statistical methods eventually resulting in a converging maximum take-off mass. In the subsequent preliminary design stage, the primary structure is distributed within the fuselage loft using knowledge based design criteria and a structural finite element (FE) model of the fuselage airframe is automatically generated. Specified load cases are evaluated and a sizing process using FSD (fully stressed design) principles is initiated to find a minimum material thickness distribution considering strength and stability criteria, thus resulting in an updated maximum takeoff mass.

This paper introduces the overall process chain as well as the data format and simulation framework. The presented work focuses on the structural aspect during the preliminary design stage of the DLR rotorcraft design process. The tools and approaches for the generation of the FE model, the calculation of the external loads, as well as the sizing methods are introduced and explained in detail. Considering novel compound configurations an outlook on structural modeling aspects is given. Finally, the paper discusses development steps for future applications and enhancements.

\section{Data Format and Process Handling}

An important aspect in the set-up of an integrated, automated tool chain is the flawless connection and communication of all contributing computational tools. The integrated tools need to interact on two levels, namely data transfer and software processes which are introduced subsequently.

\subsection{CPACS}

CPACS is used for DLR rotorcraft design activities as a common data model. It is a key component for the communication and data exchange between the individual computational tools and users. Its benefits are its hierarchical structure, easy access and readability. Since it is acting as the central component in the design chain it serves as an interface for all integrated tools $n_{t}$, thus significantly reducing the required interfaces $n_{i}$ from

$$
n_{i}=n_{t}\left(n_{t}-1\right)
$$

of a traditional approach to

$$
n_{i}=2 n_{t}
$$

as can be seen in Fig. 1 (for $n_{t} \geq 3$ ), where the blue curve shows the number of interfaces using a traditional approach (as depicted on the left) while the red curve shows the centralized CPACS approach (as illustrated in the centered figure).

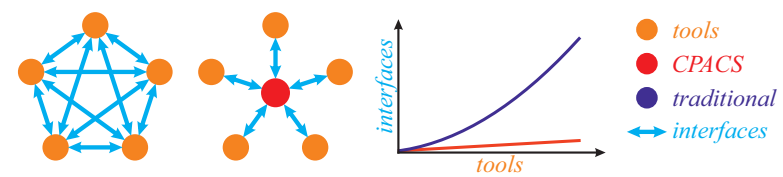

Fig. 1 Interface reduction due to CPACS 


\subsection{RCE}

The design tools do not only exchange data, they also need to interact within a design process. Since different institutions at different sites with different expertise are integrated in the presented design process, a distributed computation approach was chosen. For this purpose, the inhouse developed software RCE is used. The corresponding specialists develop and maintain their software which is installed on locally separated servers. The design tools are published to authorized partners who can execute the programs but cannot access the source code of the design tools, i.e. the knowledge of each discipline stays at the developing institute. Data is transferred via an internal network, i.e. a CPACS file is sent from the local user to the server where the desired tool is stored. The tool reads its input from this CPACS file and stores its computed output in an updated CPACS file which is then transferred back to the user.

\section{Rotorcraft Design Process}

The presented design process starts from scratch and comprises the conceptual and parts of the preliminary stage. Typically, the minimum TLARs cover payload, range, cruise speed and the rotor configuration. Currently, three types of rotor configurations are supported for automated initialization: standard, coaxial and tandem (see Fig. 2, from left to right).

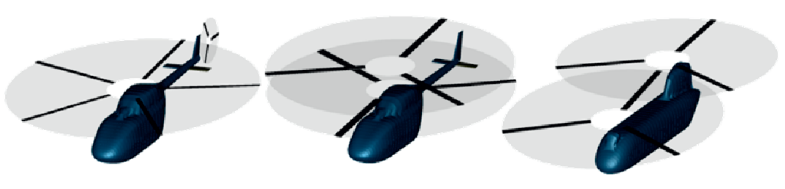

Fig. 2 Supported rotorcraft configurations

Progress in the design process leads to an increase in detail level since more input information becomes available by increasing output. Therefore, design tools can generally be classified in four levels, ranging from a coarse level 0 (L0) to a high fidelity level 3 (L3):
- LO tools mostly use empirical methods with very simple physical assumptions. They provide much output with only limited input. The objective is the generation of a first data set.

- L1 tools have a better physical modeling but are still fast enough to perform iterative procedures. Primary sizing is performed on this level. The objective is to complete the data set in order to create the first flight mechanics model.

- L2 tools feature a very good physical modeling but as a resulting disadvantage they require much computational investment, i.e. hardware and time. These tools typically comprise the preliminary design and expand the data set.

- L3 tools are the most complex design tools. They have the highest time demand and their pre- and post-processing cannot be performed automatically.

Figure 3 illustrates the global design process as applied in the presented work. Evaluating the TLARs leads to the generation of an initial model by using L0 tools which rely on a statistical data base of about 160 existing rotorcraft.

This initial model comprises a first estimation of the mass fractions resulting in a first estimation of the maximum take-off mass $m_{m t o}$ according to

$$
m_{\text {mto }}=m_{\text {oem }}+m_{\text {pay }}+m_{\text {fuel }}
$$

where

$\begin{array}{ll}m_{\text {oem }} & \text { operating empty mass (see Eq. 6) } \\ m_{\text {pay }} & \text { payload } \\ m_{\text {fuel }} & \text { fuel mass }\end{array}$

Payload is generally specified within the TLARs while the fuel mass depends on the chosen engine(s), the drag caused by the rotorcraft surfaces, and the desired range. This approach shows that for this initial estimation existing configurations are required. Novel configurations can only be roughly estimated by comparison to already existing similar rotorcraft. 


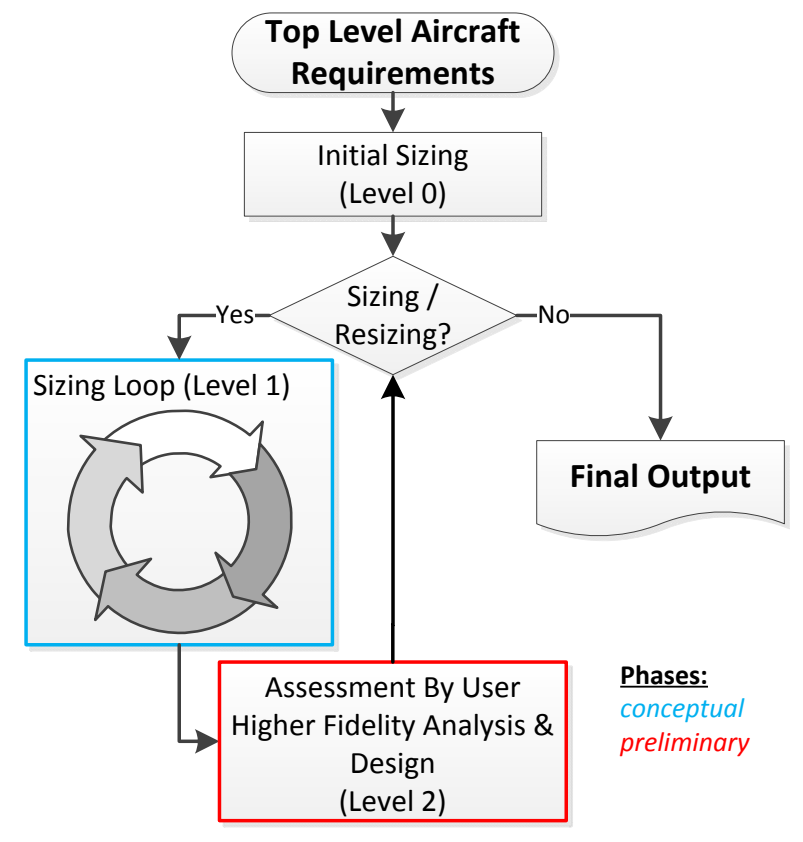

Fig. 3 Flowchart of the virtual design approach [26]

\subsection{Conceptual Design Stage}

After an initial configuration has been created, the sizing loop of Fig. 3 is initialized at the conceptual stage using L1 tools, further increasing the level of detail. Figure 4 overviews this process which is iterated until convergence of the take-off mass $m_{m t o}$ is achieved.

The calculation of the rotor characteristics is substantial for the design. A knowledge based procedure [7] is used to optimize radius, chord length, angular velocity respectively tip speed using a series of characteristic rotor parameters (aspect ratio, rotor solidity, blade loading, advance ratio, energy ratio, Lock number).

Subsequently, an outer fuselage surface is generated using a CATIA based approach [8]. This approach splits the fuselage into several segments which can individually be scaled, thus assembling the complete fuselage. Different generic templates for each fuselage segment are deposited to allow fuselage generation for each supplied configuration as specified in Fig. 2.

After the outer shape has been defined, the aerodynamic properties are calculated by a module based on the commercial software VSAERO

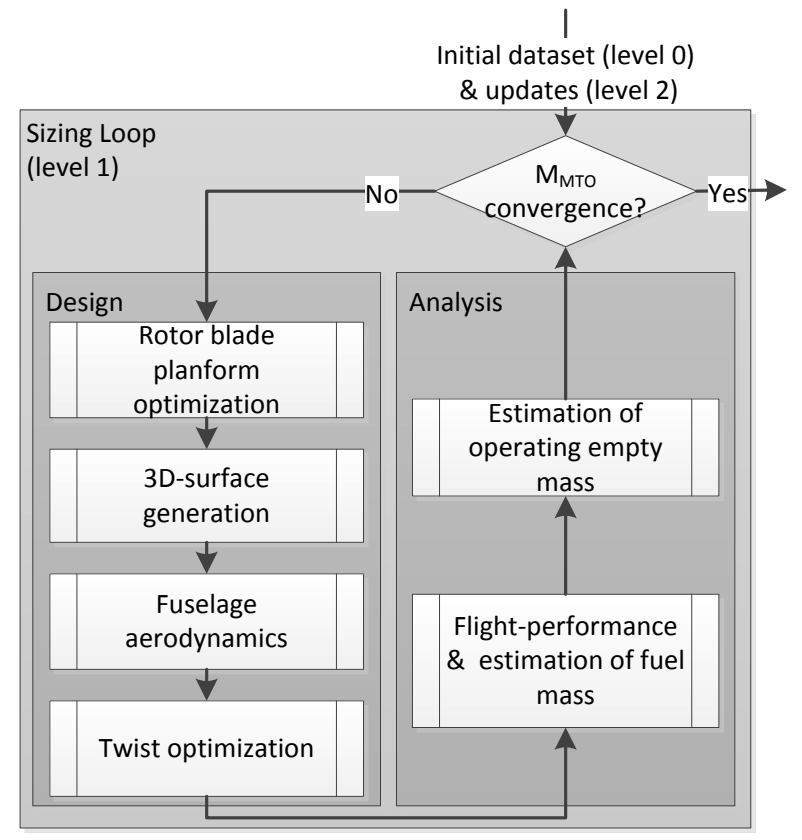

Fig. 4 Flowchart of the sizing loop [26]

[11] which uses the potential flow theory with an incompressible and inviscid approach. Compressibility corrections can be assessed by the integrated Prandtl-Glauert or Karman-Tsien approaches. Viscous effects as well as boundary layer transition and separation can be evaluated due to integration of an integral boundary layer formulation. Pressure drag is estimated based on the calculated separation line. Currently, drag influence from rotor hub, landing gears or attachments are not implemented in the drag calculation since the integrated panel methods cannot resolve it in a reliable manner. The force polars obtained by this step must be corrected to cover the influence of skids and additional attachments. The polars of the stabilizers are calculated separately.

An optimization of the blade twist can be performed in order to minimize the required power for the design flight condition. The trim calculation for this procedure is conducted by the software HOST (Helicopter Overall Simulation Tool, [2]). A linear twist distribution is iterated in order to reduce the required power for the considered flight condition.

Fuel mass is estimated in an iterative process, 
again using HOST for the trim calculation. For all flight segments, trim calculations are conducted at the beginning and at the end of every segment obtaining the required power and consequently predicting the mean fuel flow and actual range. Comparing actual and required range leads to a correction of the actual fuel until fuel mass converges and the requested range is met.

Detailed information on the aforementioned methods is given in $[7,26]$.

At this point of the design process sufficient parameters have been calculated to allow a first estimation of component masses. Statistical and empirical methods are used to break down $m_{m t o}$ into group masses, e.g. the structural, power plant, systems and furnishings group. The implemented methods are described in detail in ch. 4.

\subsection{Preliminary Design Stage}

The external configuration developed during the conceptual phase serves as starting point for the preliminary design which mainly deals with the determination of the internal configuration and more sophisticated aerodynamics, such as interactions and local flow problems. At this stage, specialists of the different disciplines design and analyze their portion of the aircraft. Sophisticated methods are applied, typically comprising L2 tools.

Currently an FE based module for structural sizing of metallic fuselages according to specified static and quasi-static flight and ground load cases is implemented in the presented design chain. This approach allows an enhanced evaluation of the fuselage weight, especially when novel designs are investigated which cannot be compared to already existing designs. A detailed description of the structural assessment is given in ch. 5.

\section{Mass estimation}

Mass estimation constitutes an essential part of the design process since the mass determines and influences many design and performance parameters. For instance does the weight determine the required lift generated by the rotor for vertical flight. The required lift then determines the dimensions of the rotor blade and the rotational velocity which in turn determine respectively influence the engine power, fuel consumption and the gear box(es).

For the mass estimation in the presented work, several estimation methods have been implemented, such as Beltramo [1], Layton [9], Palasis [14], Prouty [16], and Johnson [5].

In order to profit from a high coupling grade of geometric and performance characteristics it was decided to use the methods presented by Johnson for the component mass estimation during conceptual design. These methods are based on the U.S. Army Aeroflightdynamics Directorate (AFDD) models and feature technology factors $\chi_{i}$ which allow individual scaling according to different technology standards. For each component mass $m_{\text {comp }}$ consisting of $n$ subcomponents (e.g. the fuselage component consists of additional individual elements to account for crashworthiness, alighting gear integration, tail and/or wing folding, marinization, etc.) it is

$$
m_{\text {comp }}=\sum_{i=1}^{n} \chi_{i} m_{i}
$$

Validating the reference model (a standard configuration loosely based on an EC135) it was decided to follow the approach of Russell and Basset [18] to use one general technology factor for all components. Applying a technology factor of $\chi=0.7$ resulted in a deviation of $\Delta m_{m t o} \approx 3 \%$ between the calculated $m_{m t o}$ and the referenced $m_{m t o, r e f}$.

By summation of the individual component masses the group masses can be obtained. The empty mass $m_{e m}$ is calculated by summation of the group masses

$$
m_{e m}=m_{\text {struct }}+m_{\text {prop }}+m_{\text {sys }}+m_{f e}
$$

with the group masses

$m_{\text {struct }}$ structural mass

$m_{\text {prop }}$ propulsion (power plant) system mass

$m_{s y s} \quad$ systems mass

$m_{f e} \quad$ mass of furnishings and equipment 
Adding the mass of the operators items $m_{o i}$ to the empty mass $m_{e m}$ leads to the operating empty mass

$$
m_{o e m}=m_{e m}+m_{o i}
$$

Adding the payload and fuel mass to $m_{\text {oem }}$ results in an updated take-off mass $m_{m t o}$, thus allowing a comparison to the initial $m_{m t o, 0}$ which is used as convergence criterion of the conceptual sizing loop.

Figure 5 shows an overview of the implemented mass estimation methods applied to a generic medium-sized utility rotorcraft in standard configuration, as displayed in the graph. Since a specific engine was considered in the presented mass estimation process, the engine mass is unaffected. The methods provided by Palasis are partly those provided by Beltramo and Layton, therefore the estimated masses show the same results for certain components. Prouty does not provide an estimation method for the load handling system while Beltramo's and respectively Palasis' regression formula for this mass returns a negative value, therefore it was automatically set to zero as no reliable value was available for this system. In general, Johnson's methods appear to estimate the highest masses of all implemented methods (assuming $\chi=1.0$ ), for instance the furnishings mass is heavier than calculated with the other approaches. An explanation is that for certain components, Johnson offers mass ranges for medium- to heavy-weight rotorcraft, which are linearly interpolated by the presented module. Since the integration of certain systems is highly mission dependent, the user can provide specific input masses (in case the component mass is known or prescribed) for a more reliable mass estimation.

Comprising the operators items mass $m_{o i}=180$ $\mathrm{kg}$, a fuel mass $m_{\text {fuel }}=500 \mathrm{~kg}$ and a payload $m_{\text {pay }}=800 \mathrm{~kg}$ leads to the resulting take-off masses $m_{m t o}$ as presented in Tab. 1. The presented reference value $m_{m t o, r e f}{ }^{*}$ is the take-off mass calculated by applying Johnson's methods with an overall technology factor $\chi=0.7$ for each individual component. The maximum deviation of the other methods is $\Delta m \approx 5 \%$ indicating that

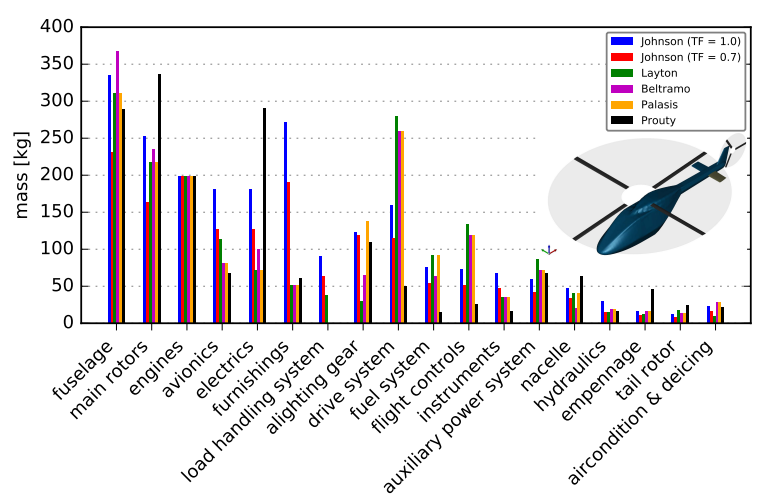

Fig. 5 Comparison of the implemented mass estimation methods

the chosen technology factor derived from the validated model marks a reliable value for this rotorcraft class.

\begin{tabular}{l||ccc} 
Method & $m_{e m}$ & $m_{m t o}$ & $\frac{m_{m t o}}{m_{m t o, r e f^{*}}}$ \\
\hline Johnson $(\chi=0.7)$ & 1,612 & 3,092 & 1.0 \\
Johnson $(\chi=1.0)$ & 2,199 & 3,679 & 1.190 \\
Beltramo & 1,746 & 3,226 & 1.043 \\
Palasis & 1,764 & 3,244 & 1.049 \\
Layton & 1,752 & 3,232 & 1.045 \\
Prouty & 1,700 & 3,180 & 1.028
\end{tabular}

Table 1 Take-off masses [kg] (rounded values)

Figure 6 shows the composition of the empty mass calculated with the methods provided by Johnson featuring a technology factor of $\chi=0.7$ for all relevant components. It can be seen that the fuselage mass constitutes the biggest share of the empty mass, thus highlighting its importance in the design process.

\section{Airframe Structural Analysis}

In order to obtain a more realistic fuselage mass estimation, L2 tools are integrated in the presented design process, designating the preliminary design stage. At the end of the conceptual design a consistent external configuration has been derived (as shown in Fig, 7), i.e. the design space for the structural members of the 


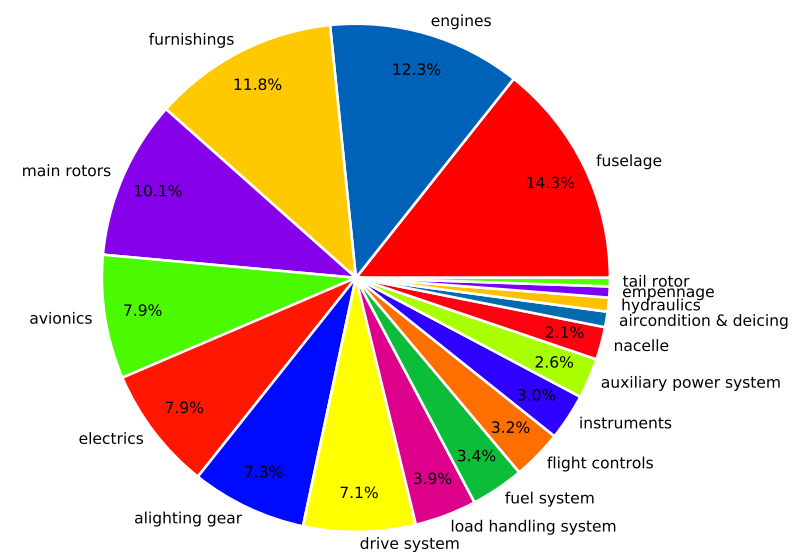

Fig. 6 Composition of $m_{e m}$ according to Johnson's methods $\left(\chi_{i}=0.7\right)$

fuselage, such as frames or stringers, is available. In a first step at preliminary level, an algorithm distributes the primary structure according to knowledge based design rules, e.g. maximum spacing between individual structural members or reinforcements around non-loadbearing cut-outs such as doors or cargo ramps [20]. Additionally, material parameters and (initial) geometric properties, such as panel thicknesses and profile dimensions, are assigned to the airframe. This information has to be specified by the user in advance to the design process. Subsequently, selected steady (nontransient) flight and ground maneuvers are evaluated and the resulting forces and moments are stored as external loads in the CPACS file. Figure 7 schematically overviews the model preparation process. It shall be noted at this point, that the presented tool is currently limited to isotropic materials, e.g. metals.

\subsection{Loads Calculation}

To calculate the external forces acting on the helicopter in static load conditions, the external forces $F_{i}$ and moments $M_{i}$ around the three mutually perpendicular axes must be in equilibrium:

$$
\begin{gathered}
\sum_{i} F_{i}=0 \\
\sum_{i} M_{i}=0
\end{gathered}
$$

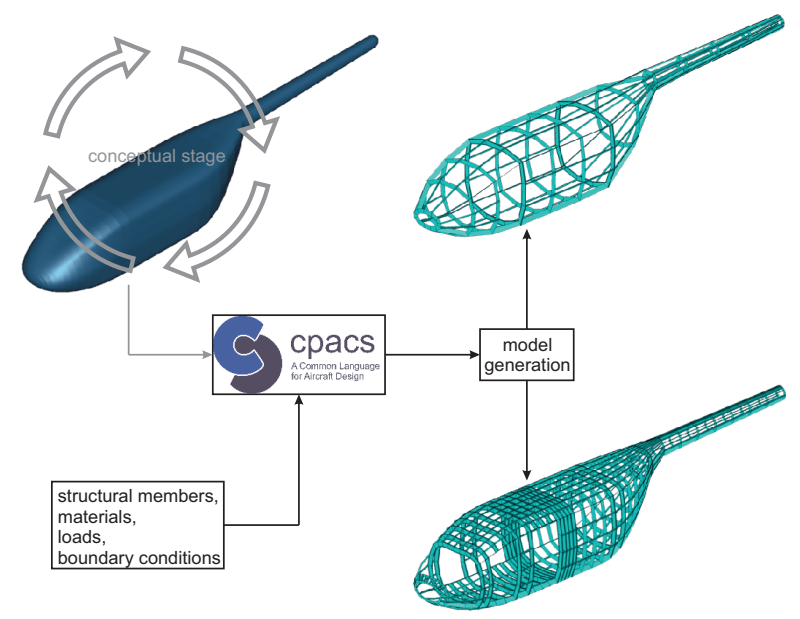

Fig. 7 Model preparation

For level flight the resultant thrust load $T$ is reacted by the weight of the helicopter, the inertial loading due to horizontal acceleration and the drag load caused by airspeed, as shown in Fig. 8 .

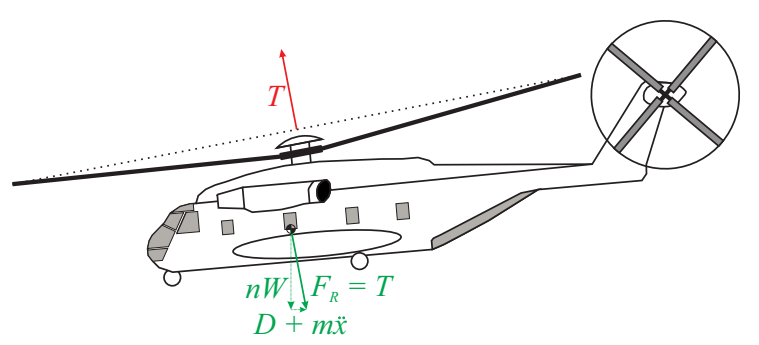

Fig. 8 Helicopter scheme for load evaluation at level flight

Thrust can be split into a propulsive force $T_{x}$ and the lift $L$ which in turn can be written due to Eq. $7 \mathrm{a}$ as

$$
T_{x}=D+m \ddot{x}
$$

and

$$
L=n W
$$

with

Weight

$n \quad$ load factor

$D \quad$ aerodynamic drag

$\ddot{x} \quad$ longitudinal acceleration 
Load factor limits are specified by EASA-CS ${ }^{1}$ 27.337 for small rotorcraft $\left(m_{\max } \leq 3175 \mathrm{~kg}\right.$ $(7000 \mathrm{lb})$ or less than nine passenger seats) and EASA-CS 29.337 for large rotorcraft. For banked turns (see Fig. 9) at constant altitude the load factor $n$ is a function of the bank angle $\phi$

$$
n=\frac{1}{\cos (\phi)}
$$

as indicated in Fig. 10.

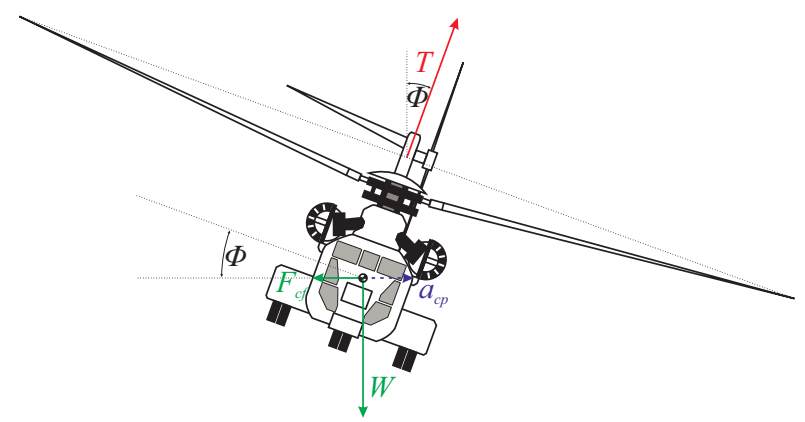

Fig. 9 Banked turn at level flight

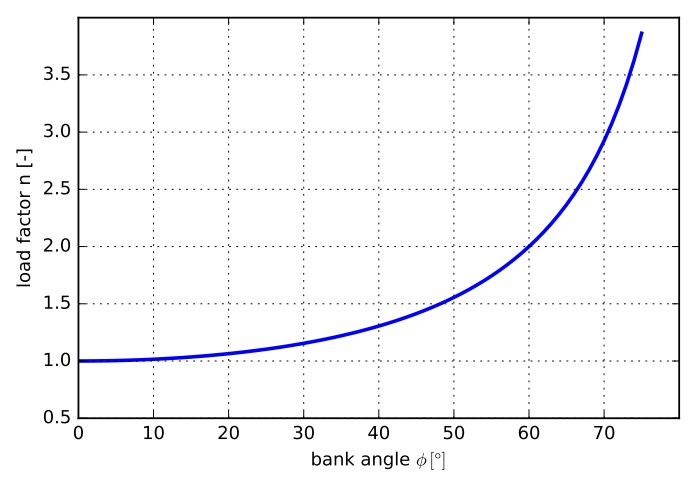

Fig. 10 Banked turn: load factor vs. angle

The centripetal acceleration $a_{c p}$ required to perform a turn with the flight path at radius $R_{t u r n}$ is

$$
a_{c p}=\frac{v^{2}}{R_{t u r n}}
$$

\footnotetext{
${ }^{1}$ Certification Specifications (CS) of the European Aviation and Space Administration (EASA)
}

where $v$ denotes the airspeed. With the relation between weight $W$, mass $m$ and acceleration $g$ under gravity

$$
W=m g
$$

and Newton's second axiom

$$
F=m a
$$

the centrifugal force $F_{c f}$ can thus be written as

$$
F_{c f}=\frac{W}{g} \frac{v^{2}}{R_{t u r n}}
$$

Helicopter parasite drag is an important aspect of performance calculation since it establishes the propulsive force and power requirement at high speed [4]. It is commonly expressed in terms of the dynamic pressure $q$ and the parasite drag area $f$

$$
D=q f
$$

with

$$
q=\frac{1}{2} \rho v^{2}
$$

where $\rho$ denotes the air density. Johnson [6] provides two possibilities to estimate the parasite drag area, either based on the maximum take-off mass $m_{m t o}^{*}$ or alternatively based on the projected area of the rotors $A_{r o t}$. For the loads calculation the weight based approach has been chosen:

$$
f=\frac{D}{q}=k\left(\frac{m_{m t o}^{*}}{1000}\right)^{2 / 3}
$$

with $k$ varying from 9 (for old helicopters) to 2.5 for current low-drag helicopters, based on historical helicopters with $m_{m t o}^{*}$ specified in pounds [lb] and $f$ given in square feet $\left[\mathrm{ft}^{2}\right]$. Torque $Q$ introduced from the main rotor into the fuselage is calculated by the relation

$$
\sum_{i} P_{i}=\omega Q
$$

where $\omega$ denotes the angular velocity of the main rotor and $\sum P_{i}$ is the total power of the main rotor which consists of several shares, e.g. induced power $P_{i}$ for thrust, $P_{0}$ to overcome blade profile drag, $P_{p}$ to overcome parasite drag and climb 
power $P_{c}$. The tail rotor force $F_{t r}$ can then be calculated by

$$
Q=F_{t r} l_{r}
$$

with $l_{r}$ being the distance between the main rotor shaft and the tail rotor shaft.

For certification all load cases that can be experienced by the rotorcraft must be considered, i.e. a changing center of gravity (COG), for instance due to fuel consumption, must be taken into account.

The rotorcraft reaction to gusts is less severe than compared to a fixed-wing aircraft. However, load factors on rotorcraft due to gusts are not insignificant. Due to the trend towards higher flight velocities gust criteria become more important as load factors generally increase with airspeed.

Due to the preliminary nature of the structural analysis, the aforementioned effects have not (yet) been taken into account.

\subsection{Model generation}

Subsequently, an FE model in GFEM (global finite element) quality is generated, as default one skin panel element comprises one stringerframe-bay. Modeling is conducted in ANSYS using APDL (ANSYS parametric design language). Engineering constants of the reinforcing profiles, such as COG, section area, and moments of inertia, are calculated by evaluating the profile dimensions and assigned to the corresponding beam elements. Frame webs and skin panels are discretized using elastic shell elements (ANSYS Shell 181) while stringers and frame flanges are modeled using beam elements (ANSYS Beam 188). Component masses estimated during the conceptual design phase are modeled as single nodal masses being coupled to the structure over a user-specified influencing region by RBE3 elements. The rotors are also discretized as nodal masses where the external forces and moments are applied. Figure 11 shows an exemplary FE model (for visual reasons only one load/mass constraint is shown) with skin panels and the reinforcing structure respectively. Cutouts for non-loadbearing elements, e.g. doors, windshield, lookouts have been applied to the air- frame using the stage modeling approach [20] which also allows a realistic termination of the stringers in the tail boom.

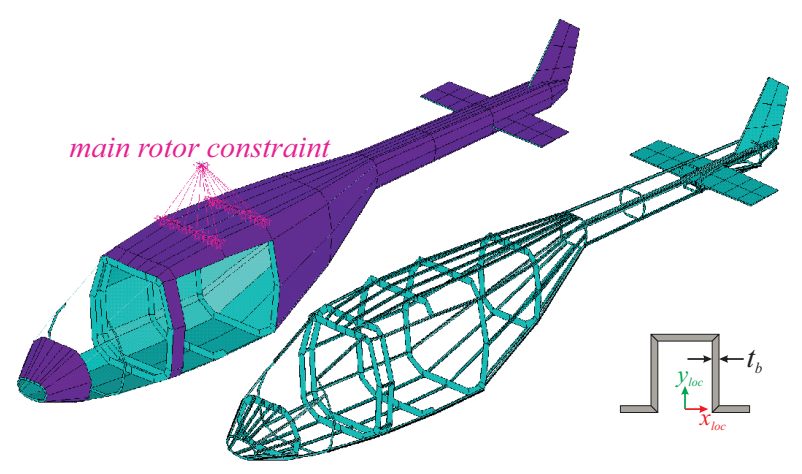

Fig. 11 FE airframe model

\subsection{Static analyses and sizing process}

Static computations are conducted using the linear-elastic ANSYS solver. Figure 12 shows a static analysis of the hovering load case, using the generic rotorcraft model as introduced in Fig. 11. The fuselage structure is made of aluminum 2024 (as specified in Tab. 2), with flat frames of different heights and thicknesses of $1.4 \leq t_{i} \leq 2.0$ [mm]. The stringers feature hat profiles (as displayed in Fig. 11, corner) with $t_{b}=1.5[\mathrm{~mm}]$ for all sheets while the skin panels feature a thickness of $t=1.6[\mathrm{~mm}]$.

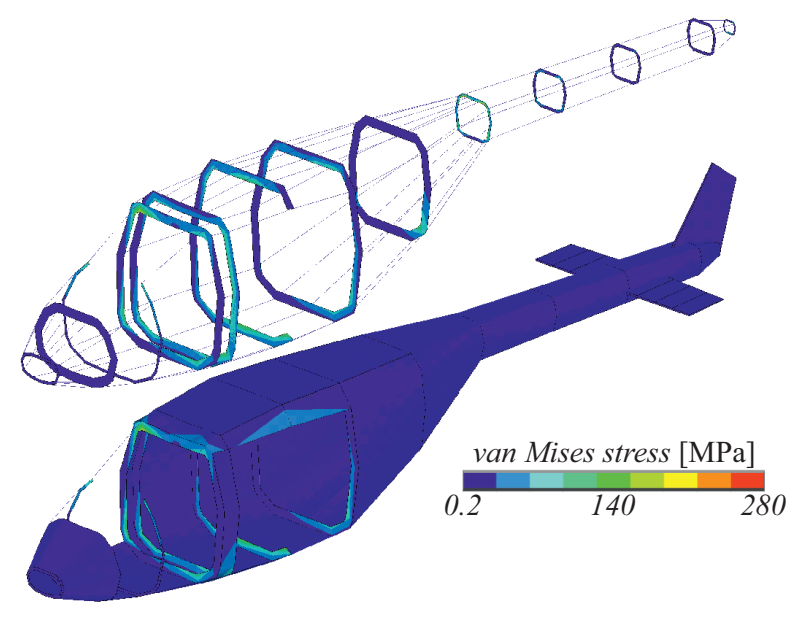

Fig. 12 Static hover analysis

It is observable that in the hovering load case 
the highest stresses arise around the cabin where the main rotor is mounted and the highest nodal masses are located, and in the first frame of tail boom due to the reduction of stringers and the lateral force introduced by the tail rotor.

$\begin{array}{lcc}\text { Young's modulus } E & 67.7 & {[\mathrm{GPa}]} \\ \text { Density } \rho & 2,800 & {\left[\mathrm{~kg} / \mathrm{m}^{3}\right]} \\ \text { Poisson's ratio } v & 0.248 & {[-]} \\ \text { Yield strength } & 320 & {[\mathrm{MPa}]}\end{array}$

Table 2 Aluminum 2024 - material properties

The sizing tool is an APDL based module, originally designed for aircraft wings [13], then adapted to aircraft fuselages [19] and finally to helicopter applications [21]. Strength evaluation is based on fully stressed design (FSD) principles. Local compressive and shear buckling methods as proposed by Bruhn [3] are implemented to guarantee sufficient safety against stability failure.

Equivalent stress $\sigma_{e q}$ is computed for all elements. The thickness of each shell element is then reduced by the factor

$$
r f=\frac{\sigma_{e q, \text { max. }}}{\sigma_{e q}}
$$

where $\sigma_{e q, \text { max. }}$ denotes the maximum allowable equivalent stress, as specified by the material or stability limits. Therefore, the thickness can be reduced as long as the allowable stress limits are not exceeded.

For the stringers the sheet thicknesses $t_{b, i}$ of all beam elements of one individual stringer are scaled equally by a common scale factor $r$ (see Fig. 11), i.e.

$$
t_{b, i}^{\prime}=r \cdot t_{b, i}
$$

while the basic cross section is maintained.

This process is repeated for each specified load case and for each element the maximum required skin respectively sheet thickness is stored and applied to the corresponding panel respectively frame or stringer element. The resulting updated stiffness distribution is then recalculated until convergence is achieved. The final thicknesses and section areas are stored back to the

\begin{tabular}{c||ccccc} 
Turn no. & $\begin{array}{c}v \\
{[k m / h]}\end{array}$ & $\begin{array}{c}r \\
{[m]}\end{array}$ & $\begin{array}{c}a_{c p} \\
{[\mathrm{~g}]}\end{array}$ & $\begin{array}{c}\alpha \\
{\left[{ }^{\circ}\right]}\end{array}$ & $\begin{array}{c}n \\
{[-]}\end{array}$ \\
\hline 01 & 200 & 500 & 0.63 & 32.2 & 1.18 \\
02 & 300 & 500 & 1.42 & 54.8 & 1.73 \\
03 & 400 & 750 & 1.68 & 59.2 & 1.95 \\
04 & 400 & 500 & 2.52 & 68.3 & 2.71
\end{tabular}

Table 3 Flight data for banked turns

CPACS file as well as the updated masses $m_{\text {struct }}$, $m_{\text {em }}, m_{\text {oem }}$ and $m_{m t o}$.

Exemplary sizing processes comprising the hovering state, cruise flight with a maximum velocity of $v_{\max }=400 \mathrm{~km} / \mathrm{h}$ and several banked turns, as specified in Tab. 3, are shown in Fig. 13. Sizing scenario no. 7 considers a $+2.5 \mathrm{~g}$ pull-up maneuver instead of the banked turn no. 4 .

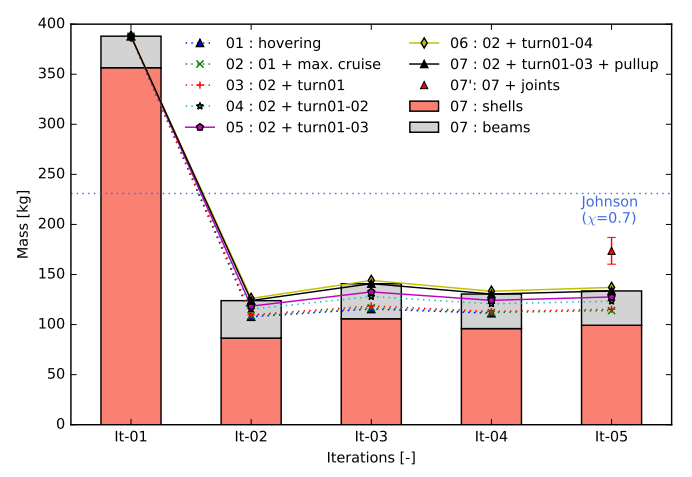

Fig. 13 Sizing process with different load case scenarios

It can be observed that the weight grows with an increase in considered load cases: The pure hovering state results in a fuselage mass of $m_{f u s} \approx$ $111 \mathrm{~kg}$ while the addition of the maximum cruise flight load case slightly increases the fuselage mass to $m_{f u s} \approx 114 \mathrm{~kg}$. The first banked turn increases the fuselage mass to $m_{f u s} \approx 115 \mathrm{~kg}$. The centrifugal load caused by this turn is comparably small since the chosen turn radius is rather large and the velocity rather slow. In contrast, the second turn scenario at high velocity increases the fuselage weight to $m_{f u s} \approx 123 \mathrm{~kg}$ due to the higher speed which increases the load factor. This effect can also be observed at turn no. 
3: Even though the radius has been increased by $\Delta r=50 \%$ to $r_{t u r n}=750 \mathrm{~m}$ the load factor increases due to the higher speed thus increasing the fuselage weight. Turn no. 4 represents a rather steep turn causing a load factor of about $n \approx 2.7$. In load scenario no. 6 it can be seen that steep turns increase fuselage weight more than the addition of a $+2.5 \mathrm{~g}$ pull-up maneuver, as shown in scenario no. 7. However, it shall be noted that this kind of maneuver is not representative for a transport, especially an emergency helicopter. Maneuver issues are of particular importance for combat helicopters which may be required to perform maneuvers consisting of high load factors and pull-ups, steep turns and rollovers.

The bars represent the share of the beam elements (representing the stringers) and the shell elements (representing the frames and the skin panels) of the sizing process comprising load case scenario no. 7 (hovering, cruise, three banked turn scenarios and a pull-up maneuver). The initial thicknesses of the shell elements were set to $t=5 \mathrm{~mm}$ to allow for a reliable convergence of the computations. The red triangle represents the sized fuselage mass of scenario no. 7 with an additional weight penalty due to joints, ranging from $20-40 \%$ of the fuselage mass.

Figure 14 shows the results of the sizing scenario no. 7, the required thicknesses and the corresponding load cases responsible for the calculated thicknesses. Note that the empennage elements were not included in the sizing process. In general, the tail boom is mainly sized due to the required turn rates about the yaw-axis, in the presented example it is the hovering load case which introduces a lateral force in the rear. The dominant load case, however, is the $+2.5 \mathrm{~g}$ pullup maneuver due to its high load factor. Most of the load is introduced in the cabin area due to the position of the main rotor and highest nodal masses. Majority of the elements is sized according to the shell buckling criterion caused by the $+2.5 \mathrm{~g}$ pull-up maneuver. The areas around the cut-outs require higher thicknesses since these regions distribute the loads around the fuselage openings (assumed that the doors must not carry any loads to prevent door frame deformation in case of emergency landings).

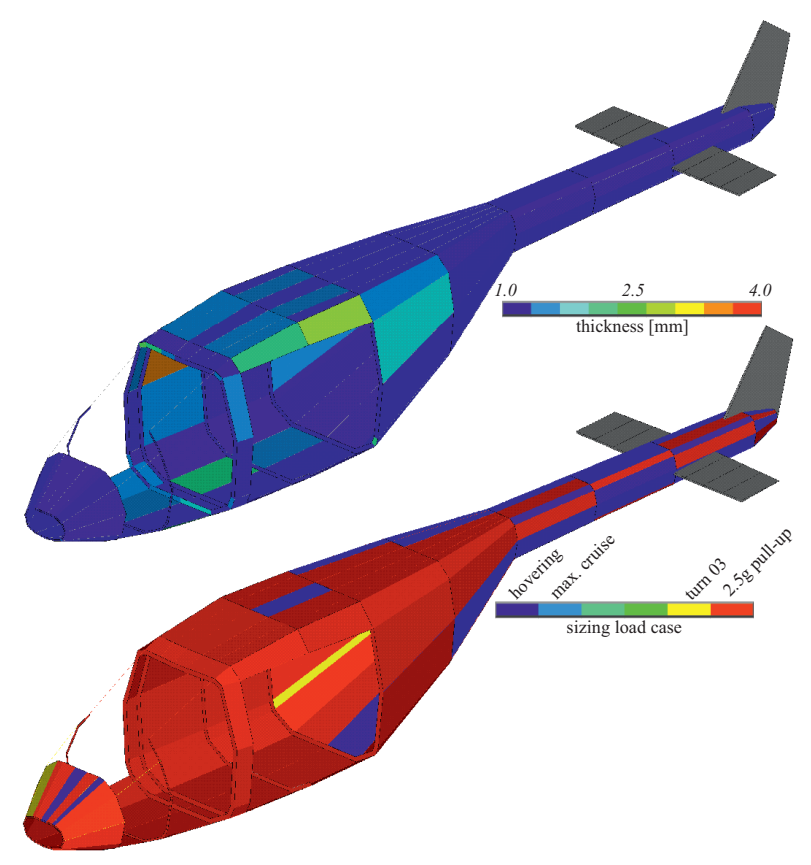

Fig. 14 Sizing results (scenario no. 7)

Comparing the FE results with the estimations based on statistics a comparably big deviation can be observed. Banked turns and pull-up maneuvers can be considered as a roughly sufficient load scenario for a first structural static sizing at early design stages. Weight penalties must be considered since the fuselage model represents preliminary detail level which lacks secondary structure, i.e. clips to connect stringers and frames, fasteners, joints, windshield and its mounting, doors and their opening mechanisms, cabin floor, etc. are not modeled. Masses of joining elements may be estimated, depending on their amount and design, to account for a weight penalty of $m_{\text {joints }} \approx 20-40 \%$ of the fuselage mass [23]. Another weight increase is seen by an extension of the integrated load cases, e.g. the touch down during landing or asymmetric flight maneuvers (combination of maneuvers like rolling pull-ups, sideslips and yaw, etc.) which result in a combination of lateral, vertical, longitudinal load factors and simultaneously rotational accelerations.

Figure 13 shows that a realistic fuselage mass es- 
timation depends on properly predicted maneuvers which highly depend on the mission profile of the rotorcraft, thus marking a frontier between statistical or analytical L1 and computational L2 methods. However, a proper prediction of helicopter maneuvers is considered an illusive goal that still challenges helicopter analysts for years to come [10].

\subsection{Lifting surfaces}

To overcome the physical limitations at high speed cruise (e.g. compression effects) of rotorcraft, novel concepts may comprise axial thrusters and (additional) lifting surfaces. These wings generate lift in horizontal flight so that the main rotor can be unloaded and slowed down. The resulting lack of thrust caused by the unloaded main rotor is then compensated by additional propulsive force generated with auxiliary propellers, thus increasing the cruise speed. In that state, wings have to carry about $75 \%$ of the rotorcraft mass.

Simple wing models have been integrated in the model generation. The wings modeled consist of their primary structure, i.e. webs, spars and the panels. Therefore, the leading and trailing edges are not taken into account for the model generation.

Wings can be integrated into the airframe either by the integration of a wingbox or by main frame attachments. However, a suitable arrangement of the gear box, the drive system, and the wing integration structure is required. Currently, wings are mounted to the fuselage structure with rigid beam elements, showing a simplified approach. Figure 15 shows this first approach to integrate wings into the rotorcraft airframe, featuring an aperture in the center for the integration of the main rotor shaft and main gear box.

\section{Conclusion and Outlook}

Within the projects RIDE and EDEN, DLR created an automated and integrated tool chain for rotorcraft design at the conceptual and preliminary design stages. Integrated tool levels range

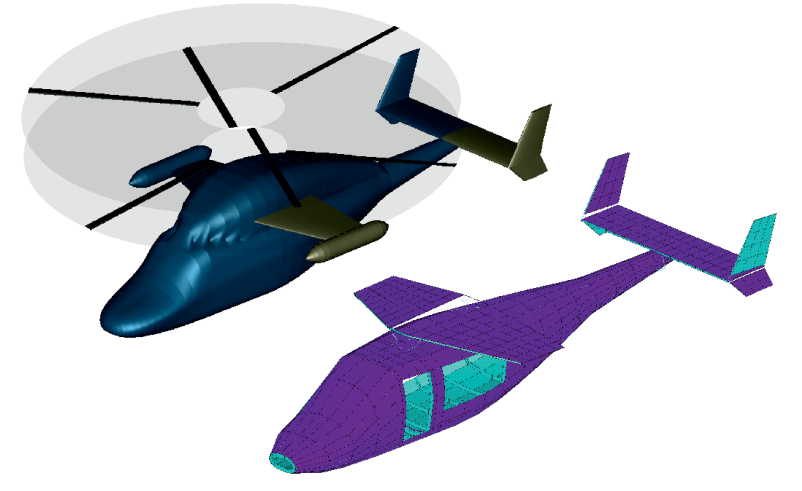

Fig. 15 Generic compound rotorcraft airframe with lifting surface

from statistics based to higher fidelity tools, e.g. based on FEM. In this paper the L1 mass estimation module and the L2 structural analysis module have been presented. For the presented medium weight utility rotorcraft, a reduction of the fuselage mass when shifting from L1 to L2 tools was observed and explained. However, novel configurations require an extension of the presented tool chain:

- The integration of wings is essential since wings are seen as a key driver in the aim to overcome physical limitations, such as cruise speed. A suitable arrangement and a detailed modeling of the wing integration structure is necessary for sufficient assessment.

- A key technology is the integration of composite materials, thus allowing to tailor the material to the load paths. Early estimations during the 1980's consider additional technology factors of about $\chi \approx 0.8$ for the fuselage weight [25] indicating a significant weight reduction compared to metallic airframes.

- The integration of crashworthiness tools may be beneficial since helicopters are statistically seen - more often exposed to dangerous situations and therefore more often involved in emergency landings compared to fixed-wing aircraft. The contradic- 
tional objectives of static sizing and energy absorption (linear elastic behavior versus non-linear compliance) is considered as a challenge.

License costs for commercial tools, especially for $\mathrm{CAD}$ and FE software, may be reduced by using open source solutions. Additionally, the renunciation of the dependence from one code increases flexibility and allows for comparative analysis without a major change of the programming code.

Currently the aforementioned tools for weight estimation and structural sizing are under reconstruction for integration into the new software environment PANDORA (Parametric Numerical Design and Optimization Routines for Aircraft, [15]), which is at present under development at the DLR Institute of Structures and Design.

Within this redesign a more detailed mass representation is intended, thus allowing a more realistic mass distribution of components, e.g. drive train, gearboxes, etc. which in turn shall result in a more realistic sizing output.

In 2018, DLR initiated the project TRIAD (Technologies for Rotorcraft in Integrated and Advanced Design, until 2020) in which the loads analysis will be transferred to the more powerful tool HOST. Another objective in this project is the extension of the aerodynamic calculation for L2 tools by calculating the interaction of the main rotor with other helicopter components and transfer this information back into the L1 sizing, similar to the structural mass.

With the aim of creating a design for a novel emergency helicopter, it is planned to combine these tasks with the design of a new, enhanced medical compartment.

\section{Acknowledgements}

The work presented in this manuscript was achieved within the DLR projects EDEN (Evaluation and Design of Novel Rotorcraft Concepts), FAST-Rescue (Fast and Silent Rescue Helicopter) and TRIAD (Technologies for Rotorcraft in Integrated and Advanced Design). The authors would like to gratefully acknowledge and appreciate the financial support.

\section{References}

[1] M.N. Beltramo and M.A. Morris. Parametric study of helicopter aircraft systems costs and weights. Technical report, NASA - National Aeronautics and Space Administration, NASACR-152315, 1980.

[2] B. Benoit, A.-M. Dequin, K. Kampa, W. von Grünhagen, P.-M. Basset, and B. Gimonet. HOST, a General Helicopter Simulation Tool for Germany and France. In American Helicopter Society $56^{\text {th }}$ Annual Forum, Virginia Beach, VA, USA, 2000.

[3] E.F. Bruhn. Analysis and Design of Flight Vehicle Structures. Tri-State Offset Company, 1973.

[4] W. Johnson. Helicopter Theory. Dover Publications, Inc., 1994.

[5] W. Johnson. NDARC - NASA Design and Analysis of Rotorcraft. Technical report, NASA - National Aeronautics and Space Administration, NASA/TP-2009-215402, 2009.

[6] W. Johnson. Rotorcraft Aeromechanics. Cambridge University Press, 2013.

[7] A. Krenik and P. Weiand. Aspects on Conceptual and Preliminary Helicopter Design. In Deutscher Luft- und Raumfahrtkongress (DLRK), Braunschweig, Germany, 2016.

[8] P. Kunze. Parametric Fuselage Geometry Generation and Aerodynamic Performance Prediction in Preliminary Rotorcraft Design. In $39^{\text {th }}$ European Rotorcraft Forum, Moscow. Russia, 2013.

[9] D.M. Layton. Introduction to Helicopter Conceptual Design, 1992.

[10] J.G. Leishman. Principles of Helicopter Aerodynamics. Cambridge University Press, 2nd edition, 2006.

[11] B. Maskew. Program VSAERO Theory Document: A Computer Program for Calculating Nonlinear Aerodynamic Characteristics of Arbitrary Configurations. Technical report, NASA, NASA-CR-4023, 1987.

[12] B. Nagel, D. Böhnke, V. Gollnick, P. Schmollgruber, A. Rizzi, G. La Rocca, and J. J. Alonso. Communication in Aircraft Design: Can We Es- 
tablish a Common Language? In $28^{\text {th }}$ International Congress of the Aeronautical Sciences, Brisbane, Australia, 2012.

[13] B. Nagel, M. Kintscher, and T. Streit. Active and Passive Structural Measures For Aeroelastic Winglet Design. In $26^{\text {th }}$ International Congress of the Aeronautical Sciences, Anchorage, AK, USA, 2008.

[14] D. Palasis. Erstellung eines Vorentwurfsverfahrens für Hubschrauber mit einer Erweiterung für das Kipprotorflugzeug. Phd thesis, Universität der Bundeswehr, München, 1992.

[15] Petsch, M. and Kohlgrüber, D. and Walther, J.-N. Development of a fully automated transport aircraft fuselage modelling and sizing tool using Python. In Deutscher Luftund Raumfahrtkongress (DLRK), Munich, Germany, 2017.

[16] R.W. Prouty. Helicopter Performance, Stability, and Control. Krieger Publishing Company, 2002.

[17] J. Roskam. Airplane Design Series. DARcorporation, 1989.

[18] C. Russell and P.-M. Basset. Conceptual Design of Environmentally Friendly Rotorcraft A Comparison of NASA and ONERA Approaches. In American Helicopter Society $71^{\text {th }}$ Annual Forum, Virginia Beach, VA, USA, 2015.

[19] J. Scherer, D. Kohlgrüber, F. Dorbath, and M. Sorour. A Finite Element Based Tool Chain for Structural Sizing of Transport Aircraft in Preliminary Aircraft Design. In Deutscher Luftund Raumfahrtkongress (DLRK), Stuttgart, Germany, 2013.

[20] D.B. Schwinn. Applied parametrized and automated airframe modeling methods in the preliminary design phase. International Journal of Modeling, Simulation, and Scientific Computing, 6(4):1550037, 2015.

[21] D.B. Schwinn, P. Weiand, and M. Schmid. Structural Analysis of a Rotorcraft Fuselage in a Multidisciplinary Environment. In NAFEMS World Congress, Stockholm, SWE, 2017.

[22] D. Seider, M. Litz, A. Schreiber, and A. Gerndt. Open Source Software Framework for Applications in Aeronautics and Space. In IEEE Aerospace Conference, Big Sky, MT, USA,
2012.

[23] F.R. Shanley. Weight-Strength Analysis Of Aircraft Structures. Dover Publications, Inc., 1960.

[24] B.G. van der Wall. Grundlagen der Hubschrauber-Aerodynamik. Springer, 2015.

[25] E. Vega. Advanced Technology Impacts on Rotorcraft Weight. Journal of the American Helicopter Society, 31(4):21-28, 1986.

[26] P. Weiand, D.B. Schwinn, M. Schmid, and M. Buchwald. A Multidisciplinary Process for Integrated Rotorcraft Design. In $43^{\text {rd }}$ European Rotorcraft Forum, Milan, Italy, 2017.

\section{Contact Author Email Address}

To contact the corresponding author please mail to: dominik.schwinn@dlr.de

\section{Copyright Statement}

The authors confirm that they, and/or their company or organization, hold copyright on all of the original material included in this paper. The authors also confirm that they have obtained permission, from the copyright holder of any third party material included in this paper, to publish it as part of their paper. The authors confirm that they give permission, or have obtained permission from the copyright holder of this paper, for the publication and distribution of this paper as part of the ICAS proceedings or as individual off-prints from the proceedings. 